\title{
Improvements in Glycemic Control in Type 2 Diabetes Patients Switched From Sulfonylurea Coadministered With Metformin to Glyburide-Metformin Tablets
}

\author{
WILLIAM DUCKWORTH, MD; MARCO MARCELLI, MD; MAUREEN PADDEN, MD; KENNETH KELLICK, PharmD; \\ TERESA DUHANCIK, PharmD; MICHELLE WILHARDT, PharmD; KEVIN COLGAN, MA, RPh; and ALICE ROMIE, PharmD
}

\begin{abstract}
OBJECTIVE: To evaluate the change in hemoglobin A1C (A1C) in patients with type 2 diabetes switched from coadministration of a sulfonylurea (SU), glyburide or glipizide, and metformin (SU+Met) to a single glyburide-metformin tablet.

METHODS: A retrospective cohort study design of patients with type 2 diabetes treated at 3 Veterans Affairs Medical Centers and 1 Department of Defense Medical Center was utilized. One hundred percent of patients receiving glyburidemetformin tablets were screened for inclusion. Patients with at least 6 months of prior $\mathrm{SU}+$ Met combination therapy and a baseline A1C measured within 35 days prior to or 3 days after switch to glyburide-metformin tablets were included. At least one documented follow-up A1C at $\geq 90$ days after the switch to glyburidemetformin was required for inclusion. Glycemic control, complications, lipid parameters, concomitant medications, and weight were analyzed prior to and following the switch to glyburide-metformin.
\end{abstract}

RESULTS: Seventy-two patient records were included after the disqualification criteria excluded 488 prospective patients. The mean age of the 72 patients was 62 years; average body mass index was $32.9 \mathrm{~kg} / \mathrm{m}^{2}$, average baseline A1C was $8.3 \%$, and the average time since diagnosis was 7.6 years. The mean reduction in A1C was $0.6 \%(P=0.002)$ at a mean follow-up of 196 days after the switch to glyburide-metformin tablets. Improvement in glycemic control was predominantly seen in patients with a baseline $\mathrm{A} 1 \mathrm{C} \geq 8 \%$ in whom a $1.3 \%$ mean reduction in $\mathrm{A} 1 \mathrm{C}$ $(P=0.0002)$ was achieved despite a lower mean final dose of glyburide.

CONCLUSION: The results of this study suggest that in type 2 diabetic patients with an $\mathrm{A} 1 \mathrm{C} \geq 8 \%$, switching from coadministration of a sulfonylurea plus metformin to combination glyburide-metformin tablets may provide an improvement in glycemic control in the range of a 1.2 to 1.4 absolute percentage point decrease in A1C. A randomized, prospective trial comparing these 2 methods of treatment is needed, however, to determine the precise effect provided by the unique formulation of glyburide in the glyburide-metformin tablet.

KEYWORDS: Type 2 diabetes, Glucovance, Glycosylated hemoglobin, A1C

J Managed Care Pharm. 2003(9)3: 256-62

\section{Authors}

WILLIAM DUCKWORTH, MD, is Director, Diabetes Research, TERESA DUHANCIK, PharmD, is a Clinical Pharmacist, and MICHELLE WILHARDT, PharmD, is a Clinical Pharmacist, Pharmacoeconomics, Carl T. Hayden VA Medical Center, Phoenix, Arizona; MARCO MARCELLI, MD, is an Associate Professor, Dept. of Medicine, Baylor College of Medicine, and a Staff Physician, Houston VA Medical Center, Texas; MAUREEN PADDEN, MD, is Faculty Development Fellow, Family Practice, Madigan Army Medical Center, Tacoma, Washington; KENNETH KELLICK, PharmD, is Clinical Pharmacy Coordinator, VA Western New York Health Care System, Buffalo, New York; KEVIN COLGAN, MA, RPh, is Vice President, Outcomes and Pharmacoeconomic Research, and ALICE ROMIE, PharmD, is a Clinical Pharmacist, EPI-Q, Inc., Oakbrook Terrace, Mllinois.

AUTHOR CORRESPONDENCE: Kevin Colgan, MA, RPh, Vice President, Outcomes and Pharmacoeconomic Research, EPI-Q, Inc., 17W727 Butterfield Rd., Suite F \& G, Oakbrook Terrace, IL 60181. Tel: (630) 889-1280; Fax: (630) 889-1284; E-mail: kevin.colgan@epi-q.com

Copyright $\odot$ 2003, Academy of Managed Care Pharmacy. All rights reserved.
7 ype 2 diabetes is a multifaceted disease with several contributing defects. As the incidence of type 2 diabetes continues to rise, ${ }^{1}$ treatments will need to target not only prevention of diabetes but also the multiple defects characteristic of this chronic metabolic disease.

Two major metabolic defects responsible for hyperglycemia include increased insulin resistance and reduced insulin secretion in response to a glucose load. Almost all patients with type 2 diabetes are resistant to insulin action, leading to overproduction of glucose by the liver and underutilization of glucose peripherally. ${ }^{2,3}$ In individuals with insulin resistance, but without type 2 diabetes, the pancreas is capable of producing sufficient amounts of insulin to overcome the insulin resistance and maintain normoglycemia. However, when pancreatic $\beta$-cell function is impaired, as it is in patients with type 2 diabetes, insulin levels are inadequate to compensate for the insulin resistance, and hyperglycemia ensues. As demonstrated in the United Kingdom Prospective Diabetes Study (UKPDS), both insulin resistance and impaired $\beta$-cell function are present at the time of diagnosis of type 2 diabetes. ${ }^{4,5}$ Specifically, newly diagnosed patients had approximately $50 \%$ of normal insulin sensitivity and a similar percentage of normal $\beta$-cell function. ${ }^{4}$

The UKPDS also showed that within 3 years of diagnosis of diabetes, approximately $50 \%$ of patients assigned to and remaining on monotherapy maintained an $\mathrm{AlC}<7 \%$, and by 9 years, fewer than $25 \%$ maintained an AlC $<7 \%$ with a single agent. ${ }^{5}$ These findings indicate that most patients will eventually require combinations of oral agents to achieve optimal glycemic control. Given that both insulin resistance and $\beta$-cell impairment exist at the time of diagnosis and that failure of monotherapy can occur within the first few years of treatment, early use of combinations of complementary agents may be more effective.

A commonly used antidiabetic combination is a sulfonylurea ([SU], an insulin secretagogue) plus metformin ([Met], an insulin sensitizer and a reducer of hepatic glucose production and intestinal glucose absorption). Glyburide-metformin, a single tablet containing both glyburide and metformin, has a unique formulation that modifies the pharmacokinetics of the glyburide component. The glyburide-metformin tablet delivers twice as much glyburide within the first 3 hours of mealtime dosing than commercially available glyburide alone. ${ }^{6}$ The tablet has been shown to be more effective in lowering both postprandial and fasting plasma glucose levels than single-agent 
therapy in patients failing diet and exercise. ${ }^{7-9}$ In addition, we have observed anecdotally significant reductions in AlC in patients switched from coadministration of a sulfonylurea and metformin to glyburide-metformin tablets in our respective practice settings. These anecdotal observations and the fact that no data were available differentiating glyburide-metformin tablets from combination therapy with a sulfonylurea plus metformin (SU+Met) delivered independently formed the impetus for this study.

The objective of our study was to investigate the change in the glycemic control achieved by switching from treatment with coadministered SU+Met tablets to glyburide-metformin tablets. This was achieved using a retrospective cohort study design and an analysis of the change in glycosylated hemoglobin before and after the switch to glyburide-metformin tablets.

\section{Methods}

A retrospective, medical record review was conducted for patients initiated on glyburide-metformin tablets between September 2000 and December 2001. The study included patients from 3 Veterans Affairs Medical Centers (VAMCs) and 1 Department of Defense Medical Center. All patients who had a prescription for glyburide-metformin tablets $(\mathrm{n}=560)$ had their medical records examined for inclusion and exclusion criteria.

Patients aged $\geq 18$ years or $\leq 80$ years with type 2 diabetes were eligible if they had received glyburide-metformin therapy for at least 90 days, had been treated with glipizide or glyburide plus metformin at least 6 months prior to switching to glyburide-metformin tablets, and did not exceed the maximum daily dose of $20 \mathrm{mg}$ of glyburide, $40 \mathrm{mg}$ of glipizide, $2,000 \mathrm{mg}$ of metformin (preswitch), or $20 \mathrm{mg} / 2,000 \mathrm{mg}$ of glyburide-metformin tablets (postswitch). AlC values must have been measured within 35 days prior to or 3 days after initiation of glyburide-metformin and at least 90 days after the switch. Patients were excluded if they had any of the following: diabetic ketoacidosis; congestive heart failure (ejection fraction $<40 \%$ ) requiring pharmacologic treatment (diuretics, digoxin, angiotensin-converting enzyme inhibitors, positive inotropes); acute or chronic metabolic acidosis; renal dysfunction (serum creatinine $\geq 1.5 \mathrm{mg} / \mathrm{dL}$ for males and $\geq 1.4 \mathrm{mg} / \mathrm{dL}$ for females) or hemodialysis; pregnancy; hypersensitivity to glyburide or metformin prior to initiation of glyburide-metformin tablets; concomitant use of either glyburide or metformin with glyburidemetformin tablets; or type 1 diabetes.

The inclusion and exclusion criteria were applied in an attempt to allow a critical time period for AlC to have stabilized preswitch and postswitch to glyburide-metformin tablets and to include only those who received treatment that was not contraindicated and at doses approved within each medication's package insert. Institutional Review Board approval was obtained from the responsible IRB at each participating site.

Seventy-two patients were identified who qualified by meeting the inclusion criteria at the Carl T. Hayden VA, Phoenix,
Arizona ( $\mathrm{n}=29)$; Houston VA ( $\mathrm{n}=14)$; Buffalo VA $(\mathrm{n}=21)$; and Madigan Army Medical Center, Tacoma, Washington $(n=8)$. These patients had their medical and pharmacy prescription records reviewed by nurses and pharmacists trained in outcomes research from an independent research organization and pharmacists and physicians at the study sites using a standard data collection form and data dictionary to assure standardized collection of data.

Data collected for analysis included baseline characteristics: age, sex, height, weight, ethnic origin, family history of diabetes, tobacco use, alcohol use, and history of stroke, hypertension, coronary artery disease, hypercholesterolemia, peripheral neuropathy, retinopathy, diabetic foot disease, and proteinuria. Laboratory findings collected during the treatment period included glycosylated hemoglobin AlC, fasting plasma glucose, blood glucose, serum creatinine, total cholesterol, low-density lipoprotein (LDL) cholesterol, high-density lipoprotein (HDL) cholesterol, triglycerides, blood urea nitrogen, aspartate aminotransferase, and C-peptide, if available. Baseline and follow-up laboratory testing was performed for each patient in the same laboratory at each specific site. Complications, the date of each complication, and the treatment for the complication were collected. A complication was defined as any event attributable to diabetes or its treatment, such as hypoglycemia or diabetic foot disease.

Medication dose, route, frequency, start date, and stop date were collected for the study medications from both the medical record and the pharmacy computer records at each site. Prescription data for coadministered sulfonylurea and metformin tablets were included for the 30-month period prior to the switch. Prescription data were included for glyburide-metformin tablets for a period of up to 19 months after the switch. This 19-month period represents the date between the first commercial use of a glyburide-metformin combination tablet and the date data collection was completed. Adherence was defined as the ratio of days of therapy supplied to the total days in the treatment period. The treatment period for the measurement of adherence was defined as the period from the index prescription fill date to run-out of days supplied in the last refilled prescription for the index drug. Other prescribed medications were recorded, including antilipemics and medications that can affect blood glucose levels.

\section{Statistical Analysis}

Analyses were conducted to compare the effects of the switch from SU+Met to glyburide-metformin tablets on AlC, body weight, lipid profile, patient adherence to the prescribed drug regimen, and concomitant medical conditions. Changes in baseline from AlC, lipid parameters, and weight were analyzed using a paired $t$ test. AlC change was also analyzed using paired $t$ tests for the following subgroups: patients not receiving insulin, patients not receiving additional adjunctive oral antidiabetic therapy, patients whose daily metformin dose did not increase after the switch, and patients not having any of the 
Improvements in Glycemic Control in Type 2 Diabetes Patients Switched

From Sulfonylurea Coadministered With Metformin to Glyburide-Metformin Tablets

\section{TABLE 1 Disqualified Glyburide-Metformin Patient Records}

\begin{tabular}{|c|c|}
\hline Reason for Exclusion & $\mathrm{n}=488(\%)$ \\
\hline Type 1 diabetes & $1(0.2)$ \\
\hline Age $>80$ years & $11(2.3)$ \\
\hline Not receiving SU+Met prior to initiation of glyburide-metformin tablets & $145(29.7)$ \\
\hline Not receiving SU+Met combination for 6 months prior to switch to glyburide-metformin tablets & $64(13.1)$ \\
\hline Receiving concomitant sulfonylurea or metformin with glyburide-metformin tablets & $9(1.8)$ \\
\hline Receiving glyburide-metformin tablets $<90$ days & $38(7.8)$ \\
\hline No baseline AlC within 35 days prior to or 3 days after switch to glyburide-metformin tablets & $66(13.5)$ \\
\hline No follow-up $\geq 90$ days after start of glyburide-metformin tablets & $73(15.0)$ \\
\hline Renal dysfunction & $7(1.4)$ \\
\hline
\end{tabular}

\section{TABLE $2 \longdiv { \text { Diabetic Patient Demographics at Baseline } }$}

\begin{tabular}{lr}
\hline Characteristic & \multicolumn{1}{c}{$\mathbf{n}(\%)$} \\
\hline Male & $70(97.2)$ \\
\hline Female & $2(2.8)$ \\
\hline Type 2 diabetes & $72(100)$ \\
\hline Ethnicity: & $52(72.2)$ \\
White & $8(11.1)$ \\
African/American & $3(4.2)$ \\
Latino/Hispanic & $9(12.5)$ \\
Other/Unknown & $69(95.8)$ \\
\hline Glyburide+metformin & $3(4.2)$ \\
\hline Glipizide+metformin & $69(17.2 \mathrm{mg})$ \\
\hline Mean glyburide dose & $3(5.0 \mathrm{mg})$ \\
\hline Mean glipizide dose & $63\left(32.9 \mathrm{~kg} / \mathrm{m}^{2}\right)$ \\
\hline Mean body mass index & $72(7.6 \mathrm{years})$ \\
\hline Mean number of years diagnosed with diabetes
\end{tabular}

aforementioned confounders. In addition, AlC changes were assessed for patients with a baseline A1C of less than $8 \%$ compared with those greater than or equal to $8 \%$ using a paired $t$ test. Eight percent was selected as the cutoff because American Diabetes Association (ADA) Clinical Practice Recommendations 2002 suggest that action be taken when AlC exceeds $8 \% .{ }^{10}$

Demographic and clinical data from the included patients were analyzed using standard descriptive analysis. Duration of glyburide-metformin treatment was calculated from the day of switch until the last refill date. Baseline doses of glyburide, glipizide, and metformin were measured at the last medical center visit prior to switch to glyburide-metformin. Daily doses of each component of glyburide-metformin were recorded at time of switch and at the last dose prescribed. The baseline dose of each component was compared to the switch dose and the dose at final follow-up using a Wilcoxon test for nonnormally distributed data in 2 related samples. Medication adherence rate was calculated by the number of days of therapy supplied divided by the number of days in treatment.

\section{Results}

Of the 560 patients screened at 3 VAMCs and 1 Department of Defense Medical Center, 72 patient records met inclusion criteria. The most common reasons for exclusion were patients not receiving SU+Met prior to the initiation of glyburide-metformin and no baseline or follow-up hemoglobin AlC measurement within the limits of the inclusion criteria (Table 1).

Those patients included in the study were predominantly white $(72.2 \%)$, male (97.2\%), with an average age of 61.9 years. Subjects were obese, on average, with a mean body mass index (BMI) of $32.9 \mathrm{~kg} / \mathrm{m}^{2}$. Patients had been diagnosed with diabetes, on average, 7.6 years prior to inclusion in the study (Table 2). Five patients received insulin at baseline (6.9\%), and 6 patients (8.3\%) were on an additional oral antidiabetes medication (Table 3).

Patients served as their own controls, having received SU+Met coadministration for at least 6 months prior to switch to glyburide-metformin tablets. The mean baseline AlC in the total population $(\mathrm{n}=72)$ was $8.3 \%( \pm \mathrm{SD} 1.7)$. The mean reduction in AlC was 0.6\% ( $P=0.002)$ with a mean follow-up of 196 days after initiation of glyburide-metformin. Mean daily doses of glyburide and metformin at baseline and at final follow-up were $17.2 \mathrm{mg}$ and $1,607 \mathrm{mg}$ and $14.7 \mathrm{mg}$ and $1,750 \mathrm{mg}$, 
Improvements in Glycemic Control in Type 2 Diabetes Patients Switched

From Sulfonylurea Coadministered With Metformin to Glyburide-Metformin Tablets

TABLE 3 Study Population Characteristics at Baseline Versus Last Follow-up After Switch

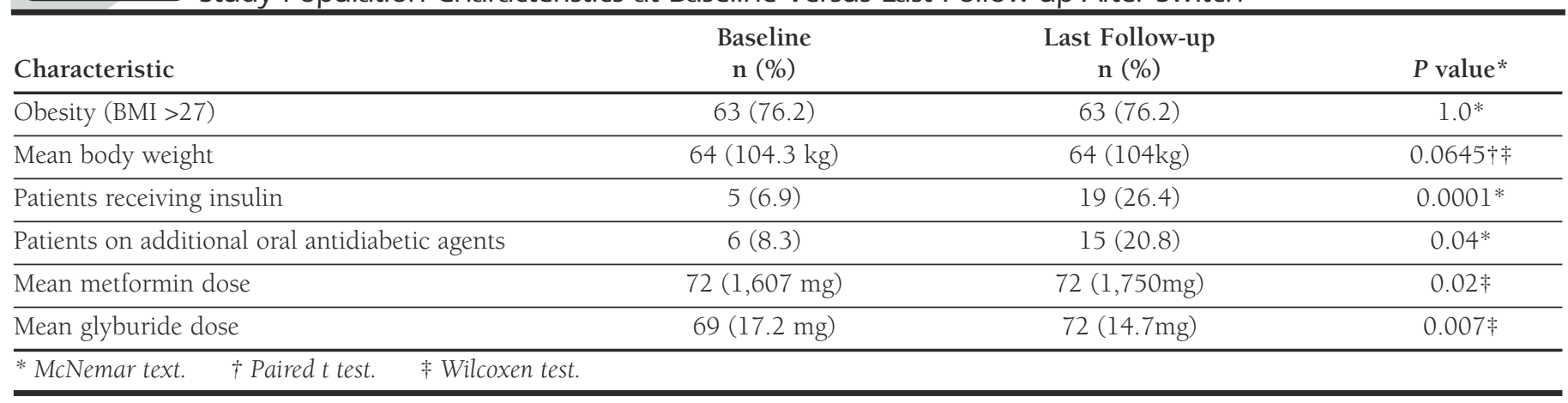

respectively. The greatest reduction in AlC was seen in those with a baseline $\mathrm{AlC} \geq 8 \%(\mathrm{n}=37)$. This cohort experienced a mean reduction in $\mathrm{AlC}$ of $1.3 \%(P=0.0002)$ with similar doses of glyburide (14.7 $\mathrm{mg}$ versus $16.9 \mathrm{mg}, P=0.077$ ) and metformin (1,743 $\mathrm{mg}$ versus $1,624 \mathrm{mg}, P=0.11)$ in both treatment periods (Figure 1).

To examine the effects of confounding variables, subset analyses were performed on patients receiving additional oral antidiabetic agents or insulin and those with an increased dose of metformin. Fifteen patients received an additional oral antidiabetic agent (i.e., thiazolidinediones, $\boldsymbol{\alpha}$-glucosidase inhibitors) either before or after the switch to glyburide-metformin. When these patients were excluded, the reductions in AlC were similar to the entire patient population regardless of baseline AlC levels ( $0.5 \%$ versus $0.6 \%$, respectively). Separate subset analyses also revealed similar mean AlC responses after exclusion of the 19 patients receiving insulin at any time during the observation period (change in AlC of $0.5 \%$ ) or after exclusion of the 16 patients whose metformin dose was increased after the switch (change in AlC of $0.5 \%$ ) (Figure 2).

Some patients had overlapping confounders (i.e. an increased metformin dose and insulin). A separate no-confounder subset analysis using paired $t$ tests was performed including only those patients who did not receive an additional oral antidiabetic agent, did not receive insulin, and did not have an increased dose of metformin after the switch to glyburidemetformin tablets. The mean baseline AlC in this cohort $(n=42)$ was $7.9 \%( \pm$ SD 1.7$)$, and the mean reduction in $\mathrm{AlC}$ was $0.4 \%$ $(P=0.35)$. Those with a baseline AlC $\geq 8 \%(n=16)$ had a mean baseline AlC of $9.7 \%( \pm S D 0.7)$ and a mean $\mathrm{AlC}$ reduction of $1.4 \% \quad(P=0.026)$. After the switch to glyburide-metformin tablets, the mean daily doses of glyburide and metformin were less than during the SU+Met coadministration period (14 mg versus $17 \mathrm{mg}$ glyburide, $P=0.003$ and $1,578 \mathrm{mg}$ versus $1,650 \mathrm{mg}$ metformin, $P=0.07)$. Those with a baseline AlC $<8 \%$ $(\mathrm{n}=26)$ had a mean baseline A1C of $6.8 \%( \pm \mathrm{SD} 1.7)$ and a mean AlC increase of $0.2 \%(P=0.14)$. This analysis reveals that the removal of confounding factors did not affect the results.

There were no significant changes in total cholesterol
(185 mg/dL before versus $178 \mathrm{mg} / \mathrm{dL}$ after, $\mathrm{n}=49$ ), HDL cholesterol (38 mg/dL before versus $39 \mathrm{mg} / \mathrm{dL}$ after, $\mathrm{n}=49$ ), LDL cholesterol (107 mg/dL before versus $96 \mathrm{mg} / \mathrm{dL}$ after, $\mathrm{n}=45$ ) or triglyceride (236 mg/dL before versus $235 \mathrm{mg} / \mathrm{dL}$ after, $\mathrm{n}=49$ ). There were also no significant differences in patient adherence to the prescribed drug regimen (92.4\% before versus $90.9 \%$ after, $\mathrm{n}=72$ ), or body weight (104.3 kg before versus $104 \mathrm{~kg}$ after, $n=64$ ). The concurrence of prescriptions for medications that may cause glucose intolerance (nicotinic acid, thiazide diuretics, or $\beta$-blockers) increased from 17 before the switch to 32 after

Six patients experienced complications related to diabetes or its treatment prior to switch, and 11 patients experienced complications after the switch to glyburide-metformin. The primary complication observed during each treatment phase was hypoglycemia. During the SU+Met treatment period, 3 patients experienced hypoglycemia in comparison to 8 patients during the glyburide-metformin treatment period. Three of 8 patients with hypoglycemia after the switch to glyburide-metformin were also receiving concomitant insulin, and the treatment for the hypoglycemia was documented as reduction in insulin dose. No patient meeting the inclusion criteria had glyburide-metformin therapy discontinued secondary to hypoglycemia.

During the SU+Met treatment period, 1 patient had a coronary artery bypass graft and 2 patients experienced diabetic foot disease. During the glyburide-metformin treatment period, 2 patients experienced chest pain and 1 patient had diabetic foot disease.

\section{Discussion}

This study found important differences between coadministered SU+Met and glyburide-metformin tablets. In patients switched to glyburide-metformin, a mean $\mathrm{AlC}$ reduction of $0.6 \%$ was realized in the total population. The improvement in glycemic control was significant predominantly in patients with an AlC $\geq 8 \%$ who experienced a mean $1.3 \%$ decrease in AlC. ADA practice recommendations indicate that an average AlC >8\% requires a change in treatment. ${ }^{10}$ The present study indicates that a significant reduction in AlC (on average 1.3\%) may be 
achieved in patients needing a change in therapy by switching patients from a SU+Met treatment regimen to glyburide-metformin tablets.

Given epidemiological findings showing that $42.2 \%$ of type 2 diabetics taking oral agents have an $\mathrm{AlC}>8 \%,{ }^{11}$ the clinical implications of these results become more important and worth studying in a prospective manner. In addition, the UKPDS of 5,102 type 2 diabetic patients showed that reducing AlC by $1 \%$ decreased the risk of any diabetes-related complication by $21 \%$, death related to diabetes by $21 \%$, myocardial infarction by $14 \%$, and microvascular complications, including retinopathy, nephropathy, and neuropathy, by $37 \%{ }^{12}$ Therefore, a reduction in $\mathrm{AlC}$ of at least 1 percentage point, as seen in patients with a baseline AlC $\geq 8 \%$ in this study, would be anticipated to impart a meaningful reduction in both cardiovascular and microvascular complications.

In a retrospective cohort study design, it is important in analysis of the findings to examine all treatment variables that may influence the study results. For example, it is necessary to consider whether changes in the doses of the components of glyburide-metformin tablets may explain the reduction of AlC after the switch. The mean daily dose of glyburide decreased and therefore would not have been expected to improve glycemic control. However, an increase in metformin dose or use of concomitant insulin or other oral antidiabetes drugs may contribute to the improvement in glycemic control.

To address these possibilities, subsets composed of patients without each confounding factor were identified and analyzed. There were 53 in the "insulin naive" subset, 57 patients in the "no other oral antidiabetic agents" subset, and 56 in the "no increase in metformin dose" subset. Based on these subset analyses, the reductions in AlC could not be attributed to increased use of concomitant insulin or oral antidiabetic drugs or to any increase in the daily dose of metformin $(0.5 \%$ for each subset versus $0.6 \%$ study population). In addition, there were no significant changes in other potentially confounding factors, including medication refill rate, body weight, or lipid levels. The greater AlC reduction also could not be attributed to decreased use of medications that may increase glucose intolerance, such as nicotinic acid, thiazide diuretics, and beta adrenergic blocking agents; instead, use of these medications increased after the switch. Thus, the variable most likely to account for the reduction in $\mathrm{AlC}$ was the actual change in treatment from coadministration of a sulfonylurea plus metformin to the glyburide-metformin tablets.

In interpreting these data, one must be aware of the potential phenomenon of regression to the mean in $\mathrm{AlC}$ measurement. Correlation between the pre-glyburide-metformin and post-glyburide-metformin readings was significant (Pearson's $r=0.5, P=0.000009$ ), suggesting that regression to the mean could only account for a portion of the difference seen. However, without a control group, it is difficult to assess the true effect of regression to the mean.
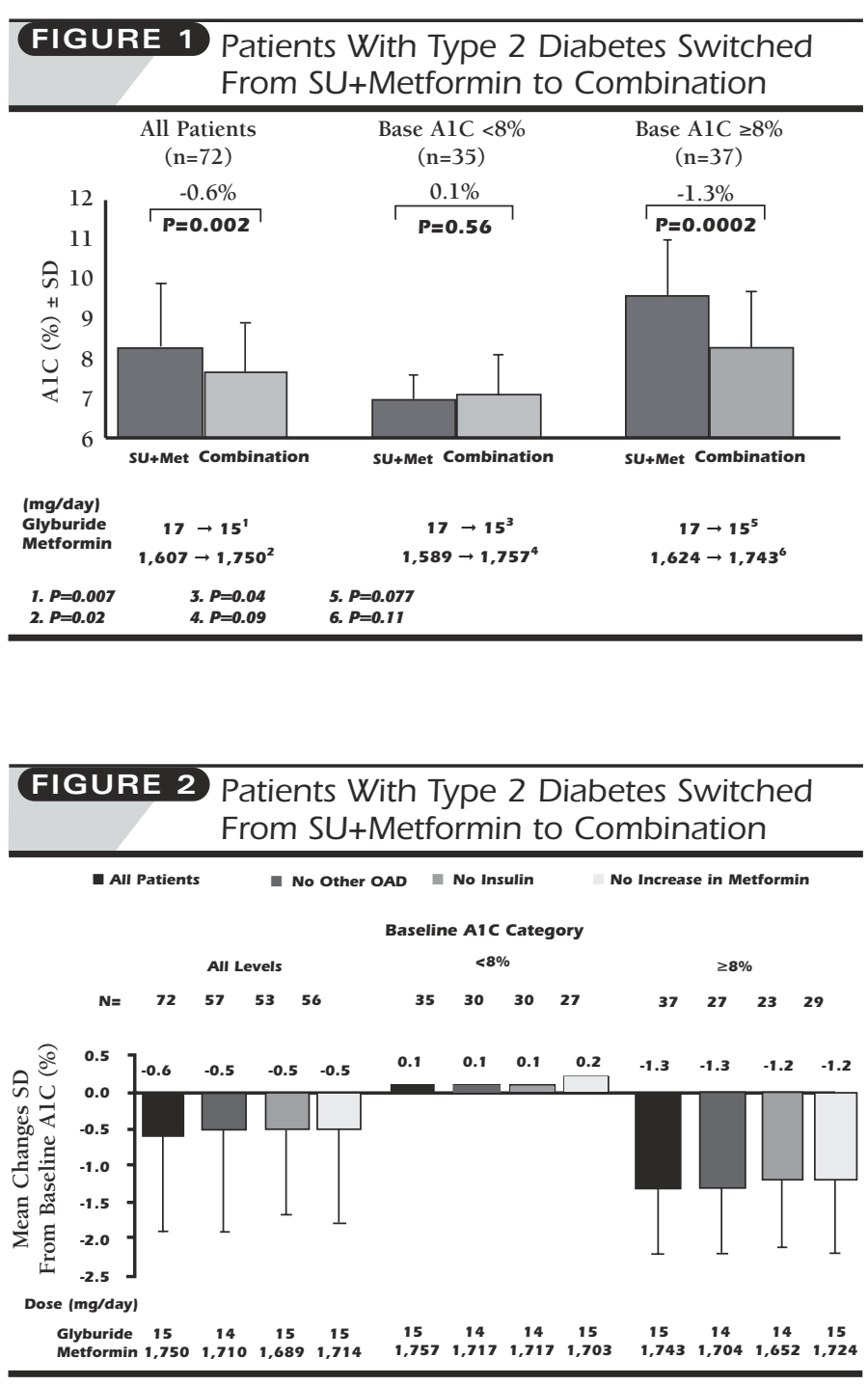

Improved glycemic control attained by switching from coadministration of the individual agents to the glyburide-metformin tablets may be attributable, at least in part, to the unique glyburide formulation of the glyburide-metformin tablets. Glyburide-metformin tablets are engineered to contain a spectrum of glyburide particle sizes. Theoretically, the smaller particles would dissolve faster and be absorbed more quickly than the larger particles. In a recent study of type 2 diabetic patients, glyburide-metformin tablets were compared to coadministration of glyburide and metformin. Results indicate that twice as much glyburide was delivered within the first 3 hours after dosing with the glyburide-metformin tablet $\left(\mathrm{AUC}_{0.3} 47 \mathrm{ng} \cdot \mathrm{hr} / \mathrm{mL}\right.$ versus $95 \mathrm{ng} . \mathrm{hr} / \mathrm{mL}$ ), while the metformin pharmacokinetic profiles were indistinguishable. ${ }^{6}$ Because glyburide-metformin tablets are routinely dosed with a meal, one may expect that the early rise in glyburide concentrations would deliver the highest 
insulin levels during the time of peak postprandial hyperglycemia. Therefore, the unique glyburide pharmacokinetic profile of the glyburide-metformin tablet may explain, in part, its improved control of hyperglycemia in the postprandial state when compared to monotherapy with glyburide or metformin. ${ }^{79,13}$

Although not powered on this specific endpoint, adherence to drug regimens before and after switch in therapy was not significantly different $(92.4 \%$ versus $90.9 \%$ ) and was not related to mean reductions in AlC. In the VA and Department of Defense settings, as well as in the private sector, beneficiaries are charged a copayment per prescription filled, typically $\$ 7$ per 30-day supply in the VAMC setting and $\$ 8$ to $\$ 35$ per 30-day supply in the private sector, depending on the copay tier in the managed care plan. With the switch to glyburide-metformin combination, only 1 prescription copayment would be required, saving the patient an additional copayment. In addition, another published study has shown that reducing the tablet number for each dosing interval improves adherence in the diabetic patient. ${ }^{14}$

The VAMCs and Department of Defense Medical Center provided an ideal setting for this retrospective study of type 2 diabetes. Diabetes care is provided longitudinally in a closed setting. Patients are seen by the same physicians and prescriptions are filled by the same pharmacy, allowing easy tracking of the effect of each treatment on the outcome of the patient. A retrospective study does not approach the rigor of a controlled, randomized trial but does provide insight into real-world outcomes in actual clinical practice.

Improved glycemic control is often achieved by stricter adherence to diet, increased exercise, medication titration, or a blend of these methods. Switching from single-dose tablets to a combination product may not be the customary course used to improve glycemic control when a patient is at the take-action threshold (AlC $\geq 8 \%$ ) published in 2000 ADA standards. ${ }^{10}$ Upward titration of one or both medications or the addition of another agent is often chosen until the patient achieves that targeted response. The results of this study suggest that the use of glyburide-metformin combination tablets can be an effective alternative to traditional medication titration in type 2 diabetic patients.

\section{Limitations}

The primary limitation of this study is the relatively small sample size. The number of cases excluded due to the absence of a baseline AlC (66 of 560 cases screened, or 11.5\%) or the use of a glyburide or metformin dose that was greater than the recommended package insert dose (50 of 560 cases screened, or $8.9 \%$ ) were major factors limiting our sample size. The sample size may have been insufficient in each subset to determine a difference in the preswitch and postswitch doses of glyburide and metformin.

The retrospective design of our study and the inclusion/exclusion criteria did not allow for control of all confounding variables, such as the daily dose of glyburide-met- formin and the utilization of insulin or other oral antidiabetic agents. The predominant glyburide-metformin effect was found in patients with a baseline $\mathrm{AlC} \geq 8 \%$. Of those with no confounders, only 16 patients had a baseline $\mathrm{AlC} \geq 8 \%$, although their outcome was similar to the study population (AlC reduction of $1.4 \%$ in the no-confounder population versus $1.3 \%$ in the study population). Further research could focus on patients whose $\mathrm{AlC}$ is $\geq 8 \%$ to evaluate the glyburide-metformin effect in a larger sample.

Due to the study being performed in the VA and Department of Defense medical centers, it is difficult to determine if the results of this study can be generalized to a more diverse population. The study population was predominantly male (97.2\%). The sample size was also not large enough to evaluate the affect of age or disease duration on outcome.

\section{Conclusion}

Patients switched from coadministered SU+Met to combination glyburide-metformin tablets experienced an overall mean decrease of $0.6 \%$ in hemoglobin AlC $(P=0.002)$ and a mean reduction of $1.3 \%(P=0.0002)$ in those patients with baseline AlC $>8 \%$. Based on the recent demonstration that the glyburide-metformin tablet delivers twice as much glyburide during the first 3 hours than does glyburide coadministered with metformin, the unique tablet design may explain, in part, the greater decrease in AlC observed in this study. Although a randomized, prospective trial is needed for verification, the present findings suggest that switching from a sulfonylurea coadministered with metformin to the glyburide-metformin tablet may improve glycemic control in patients with type 2 diabetes and an $\mathrm{AlC}>8 \%$.

\section{ACKNOWLEDGMENTS}

The authors acknowledge Farid Roman, MD, who worked with Dr. Marco Marcelli, MD, at the VA Hospital in Houston; Larry Greene, PharmD, CDE, for providing assistance with data collection at Madigan Army Medical Center; and Alicia Shillington, RN, MPH, and Mark Jewell, PhD, for providing assistance with statistical analyses.

\section{DISCLOSURES}

Funding for this research was provided by a grant from Bristol-Myers Squibb that was obtained by author Kevin Colgan. Author Alice Romie was the project manager of this research project, and author William Duckworth served as principal author of this study. Duckworth is an advisor to Bristol-Myers Squibb. Study concept and design were contributed by Duckworth, Colgan, Romie, authors Marco Marcelli, Maureen Padden, Kenneth Kellick, Teresa Duhancik, and Michelle Wilhardt and Farid Roman. Analysis and interpretation of data and drafting of the manuscript were primarily the work of Colgan and Romie. Critical revision of the manuscript was the work of Duckworth, Marcelli, Roman, Padden, Kellick, Duhancik, and Wilhardt.

The opinions or assertions contained in this article are the private views of the authors and are not to be construed as official or reflecting the view of the Department of the Army or the Department of Defense.

\section{REFERENCES}

1. Boyle JP, Honeycutt AA, Venkat Narayan KM, et al. Projection of diabetes burden through 2050. Impact of changing demography and disease preva- 
Improvements in Glycemic Control in Type 2 Diabetes Patients Switched

From Sulfonylurea Coadministered With Metformin to Glyburide-Metformin Tablets

lence in the US. Diabetes Care. 2001:24:1936-40.

2. Fujimoto, W. The importance of the insulin resistance in the pathogenesis of type 2 diabetes mellitus. Am J Med. 2000;108(suppl 6a):9S-14S.

3. Kahn, S. The importance of the B-cell in the pathogenesis of type 2 diabetes mellitus. Am J Med. 2000;108(suppl 6a):2S-8S.

4. U.K. Prospective Diabetes Study 16: overview of 6 years' therapy of type II diabetes: a progressive disease. U.K. Prospective Diabetes Study Group. Diabetes. 1996;44(11):1249-58.

5. Turner RC, Cull CA, Frighi V, Rury R. Glycemic control with diet, sulfonylurea, metformin, or insulin in patients with type 2 diabetes mellitus: progressive requirement for multiple therapies (UKPDS 49). JAMA. 1999;281:2005-12.

6. Donahue SR, Turner K, Patel S. Pharmacokinetics and pharmacodynamics of glyburide-metformin tablets (Glucovance trademark) versus equivalent doses of glyburide plus metformin in patients with type 2 diabetes. Clin Pharmacokinet. 2002;41:1301-09.

7. Garber AJ, Larsen J, Schneider SH, Piper BA, Henry D. Simultaneous glyburide-metformin therapy is superior to component monotherapy as an initial pharmacological treatment for type 2 diabetes. Diabetes Obes Metab. 2002; (4)1-8.

8. Garber AJ, Bruce S, Fiedorek FT. Durability of efficacy and long-term safety profile of glyburide-metformin tablets in patients with type 2 diabetes mellitus: an open-label extension study. Clin Ther. 2002;24(9):1401-13.
9. Garber AJ, Bruce S, Park J-S. Enhanced efficacy of glyburide-metformin combination versus monotherapy for patients with type 2 diabetes [abstract]. Endocr Pract. 2002;8:149.

10. American Diabetes Association. Standards of medical care for patients with diabetes mellitus. Diabetes Care. 2000;25(suppl 1):S33-S49.

11. Harris MI, Eastman RC, Cowie CC, Flegal KM, Eberhardt MS. Racial and ethnic differences in glycemic control of adults with type 2 diabetes. Diabetes Care. 1999;22:403-08

12. Stratton IM, Adler AI, Neil HA, et al. Association of glycaemia with macrovascular and microvascular complications of type 2 diabetes (UKPDS 35): prospective observational study. BMJ. 2002;321:405-12.

13. Blonde L, Rosenstock J, Mooradian AD, Piper BA, Henry D. Glyburidemetformin combination product is safe and efficacious in patients with type 2 diabetes failing sulfonylurea therapy. Diabetes Obes Metab. 2002;4(6):368-75.

14. Melikian C, White TJ, Vanderplas A, Dezii CM, Chang E. Adherence to oral antidiabetic therapy in a managed care organization: a comparison of monotherapy, combination therapy, and fixed-dose combination therapy. Clin Ther. 2002;24:460-67. 